\title{
Outcomes and Recommendations of an Indian Expert Panel for Improved Practice in Controlled Ovarian Stimulation for Assisted Reproductive Technology
}

\author{
Baiju Ahemmed, ${ }^{1}$ Vani Sundarapandian, ${ }^{2}$ Rohit Gutgutia, ${ }^{3}$ Sathya Balasubramanyam, \\ Richa Jagtap, ${ }^{5}$ Reeta Biliangady, ${ }^{6}$ Priti Gupta, ${ }^{7}$ Sachin Jadhav, ${ }^{8}$ \\ Ruma Satwik, ${ }^{9}$ Pavitra Raj Dewda, ${ }^{10}$ Priti Thakor, ${ }^{10}$ and Sandro C. Esteves ${ }^{11}$ \\ ${ }^{1}$ NCARE Group of IVF Centres, Kerala, India \\ ${ }^{2}$ Jananam Fertility Center, Chennai, India \\ ${ }^{3}$ Nova IVI Fertility, Kolkata, India \\ ${ }^{4}$ Cloud 9 Hospitals, Chennai, India \\ ${ }^{5}$ Nova IVI Fertility, Mumbai, India \\ ${ }^{6}$ Cloud 9 Fertility, Bengaluru, India \\ ${ }^{7}$ Fertility and IVF-Jaipur Golden Hospital \& Gupta Maternity Home, Delhi, India \\ ${ }^{8}$ Gupte Hospital, Pune, India \\ ${ }^{9}$ Centre of IVF and Human Reproduction, Sir Ganga Ram Hospital, New Delhi, India \\ ${ }^{10}$ Medical Affairs, Merck Biopharma, Mumbai, India \\ ${ }^{11}$ ANDROFERT, Andrology and Human Reproduction Clinic, Referral Center for Male Reproduction, Campinas, SP, Brazil
}

Correspondence should be addressed to Pavitra Raj Dewda; drpavitra@gmail.com

Received 17 August 2016; Revised 8 December 2016; Accepted 21 December 2016; Published 26 January 2017

Academic Editor: Samir Hamamah

Copyright (C) 2017 Baiju Ahemmed et al. This is an open access article distributed under the Creative Commons Attribution License, which permits unrestricted use, distribution, and reproduction in any medium, provided the original work is properly cited.

\begin{abstract}
Purpose. To improve success of in vitro fertilization (IVF), assisted reproductive technology (ART) experts addressed four questions. What is optimum oocytes number leading to highest live birth rate (LBR)? Are cohort size and embryo quality correlated? Does gonadotropin type affect oocyte yield? Should "freeze-all" policy be adopted in cycles with progesterone $>1.5 \mathrm{ng} / \mathrm{mL}$ on day of human chorionic gonadotropin (hCG) administration? Methods. Electronic database search included ten studies on which panel gave opinions for improving current practice in controlled ovarian stimulation for ART. Results. Strong association existed between retrieved oocytes number (RON) and LBRs. RON impacted likelihood of ovarian hyperstimulation syndrome (OHSS). Embryo euploidy decreased with age, not with cohort size. Progesterone $>1.5 \mathrm{ng} / \mathrm{dL}$ did not impair cycle outcomes in patients with high cohorts and showed disparate results on day of hCG administration. Conclusions. Ovarian stimulation should be designed to retrieve 10-15 oocytes/treatment. Accurate dosage, gonadotropin type, should be selected as per prediction markers of ovarian response. Gonadotropin-releasing hormone (GnRH) antagonist based protocols are advised to avoid OHSS. Cumulative pregnancy rate was most relevant pregnancy endpoint in ART. Cycles with serum progesterone $\geq 1.5 \mathrm{ng} / \mathrm{dL}$ on day of hCG administration should not adopt "freeze-all" policy. Further research is needed due to lack of data availability on progesterone threshold or index.
\end{abstract}

\section{Introduction}

Infertility constitutes a major health problem across all ages in India. As a result, the demand for medically assisted modalities to alleviate infertility is growing [1-3]. Since the birth of first baby through natural in vitro fertilization (IVF) in 1978, more than 5 million births have taken place worldwide through assisted reproductive technology (ART) till date $[2,4]$. Given that oocytes are the raw material to be fertilized and generate embryos, which shall be available 
for transfer or cryopreservation, an optimal number of oocytes must be obtained at the end of ovarian stimulation, below and above which, outcomes may be compromised [5]. Interestingly, in 1980, Edwards reported one clinical pregnancy per 11 oocytes retrieved, and one live birth per 15 oocytes retrieved [6]. Owing to the developments in ART in successive years, higher pregnancy rates are reported nowadays but the efficiency of oocyte utilization has not improved significantly [7]. Due to relatively low efficiency of ART, controlled ovarian hyperstimulation $(\mathrm{COH})$ with gonadotropins has been an integral element of treatment and is used to promote multiple follicular growths [8]. $\mathrm{COH}$ is used to obtain an increased number of oocytes but a consensus on optimum number of oocytes to be retrieved for a better live birth rate (LBR) is not yet clear [9]. Moreover, the impact of number (cohort size) of oocytes on the quality of embryo is crucial to know.

The ability of the oocyte to be fertilized is largely determined by the morphological and functional changes that are caused by hormonal events, specifically the fluctuation of gonadotropin hormones. Follicle-stimulating hormone (FSH) and luteinizing hormone (LH) work in a complementary manner to regulate the follicle that lead to a synergistic action of stimulating follicular growth and ovulation [10]. However, there is a lack of precision on the effect of different gonadotropins on oocyte yield in IVF.

Many studies have reported an elevated serum progesterone (P) levels above a threshold level of $>1.5 \mathrm{ng} / \mathrm{mL}$ on the day of human chorionic gonadotropin (hCG) administration $[11,12]$. Conflicting views have emerged on the rise of serum $\mathrm{P}$ levels with some researchers reporting that the raised levels might be detrimental and thus the embryos should be frozen ("freeze all") whereas others suggest that elevated P levels are associated with a good ovarian response $[13,14]$. In a prospective, randomized trial conducted by Smitz and coworkers, higher $\mathrm{P}$ levels with recombinant FSH (rFSH) compared with highly purified menotropin (HP-hMG) even after adjusting for ovarian response were observed [15]. In another randomized, open-labeled, noninferiority trial, Devroey et al. found almost similar levels of $\mathrm{P}$ with both rFSH and HP-hMG [16]. Given the nonuniform sensitivity of $\mathrm{P}$ measurement assays, especially in the low range of $\mathrm{P}$, there is a lack of clarity among IVF specialists regarding adopting "freeze-all" policy in all cycles with $\mathrm{P}$ elevation on day of hCG administration based on a single cut-off point, namely, $1.5 \mathrm{ng} / \mathrm{mL}$. Notwithstanding, among the techniques to measure the $\mathrm{P}$ levels during stimulation, isotope dilutiongas chromatograph/mass spectrometry (ID-GC/MS) is a sensitive measurement technique [17].

Evidence from outside India has been recently published to answer questions regarding the optimum number of retrieved oocytes to increase likelihood of LBR with fewer complications, the effect of cohort size on embryo quality, impact of different gonadotropins on oocyte yield, and $\mathrm{P}$ levels on the day of hCG administration on the chances of pregnancy. However, a single source that could guide the practitioners in the field of IVF related-infertility on contemporary topics is not available. Thus, the experts in the field of IVF with the help of an International moderator formed a panel to discuss these issues of high clinical relevance based on current literature and their own experiences. This article presents the outcomes and recommendations of the expert panel on these IVF related clinically relevant issues.

\section{Materials and Methods}

Nine Indian infertility experts having at least 10 years of clinical experience and academic contribution (defined by scholar contributions including peer-reviewed articles, book chapters, conference papers, and/or teaching activities) and one international moderator having over 20 years of experience were invited and accepted the task of forming a panel gathered in April 2015, to discuss important clinical issues to IVF practitioners. Among many important issues, the issues considered most pertinent to practitioners were identified a priori based on a survey among panelists. Four clinical questions have been elaborated a priori based on consensus among participants to be discussed in the expert panel meeting. (i) What is the optimum number of oocytes that is associated with the highest LBR? (ii) Is there a correlation between cohort size and embryo quality? (iii) Does the choice of gonadotropins affect oocyte yield in IVF? (iv) Should "freeze-all" policy be adopted in all cycles with $\mathrm{P}$ levels $>1.5 \mathrm{ng} / \mathrm{mL}$ on the day of hCG administration?

In preparation, an extensive literature search with keywords as "oocytes", "live birth rate", "assisted reproductive technology", "in vitro fertilization", "intracytoplasmic sperm injection", "recombinant FSH", "progesterone levels", and "gonadotropin" was performed in MEDLINE, Cochrane Library, and ScienceDirect databases from January 2011 to April 2015 to identify relevant articles to respond to the clinical questions. The keywords were provided by the moderator and expert panel and the literature search was conducted by the medical writer. Full articles published in English and in peer-reviewed and indexed journals were selected. The moderator independently screened the studies for inclusion. All the potentially relevant articles that could answer the relevant questions were examined, and these were subsequently screened by other reviewers. Discrepancies, if any, were resolved by discussion among the panel members.

\section{Results}

The search retrieved 202 citations, of which 25 were considered for full-text screening and 10 were selected for discussion by the panel (Figure 1). The questions were finalized or decided on the basis of problem/difficulty/confusion faced by the IVF experts while performing IVF/ARTs practically. Excluded articles had poorly defined methodology and/or conflicting or noncommittal results that did not allow answering the proposed clinical questions. In addition, three studies on the influence of $\mathrm{P}$ levels on the probability of pregnancy in IVF were excluded due to (i) utilization of $\mathrm{P}$ measurement in $\mathrm{nmol} / \mathrm{L}$ and cut-off levels other than $1.5 \mathrm{ng} / \mathrm{mL}$ (or $4.77 \mathrm{nmol} / \mathrm{L}$ ) [18, 19] and (ii) P determination on a day other than the day of hCG administration [20]. The attributes of the included articles are tabulated in Table 1. 


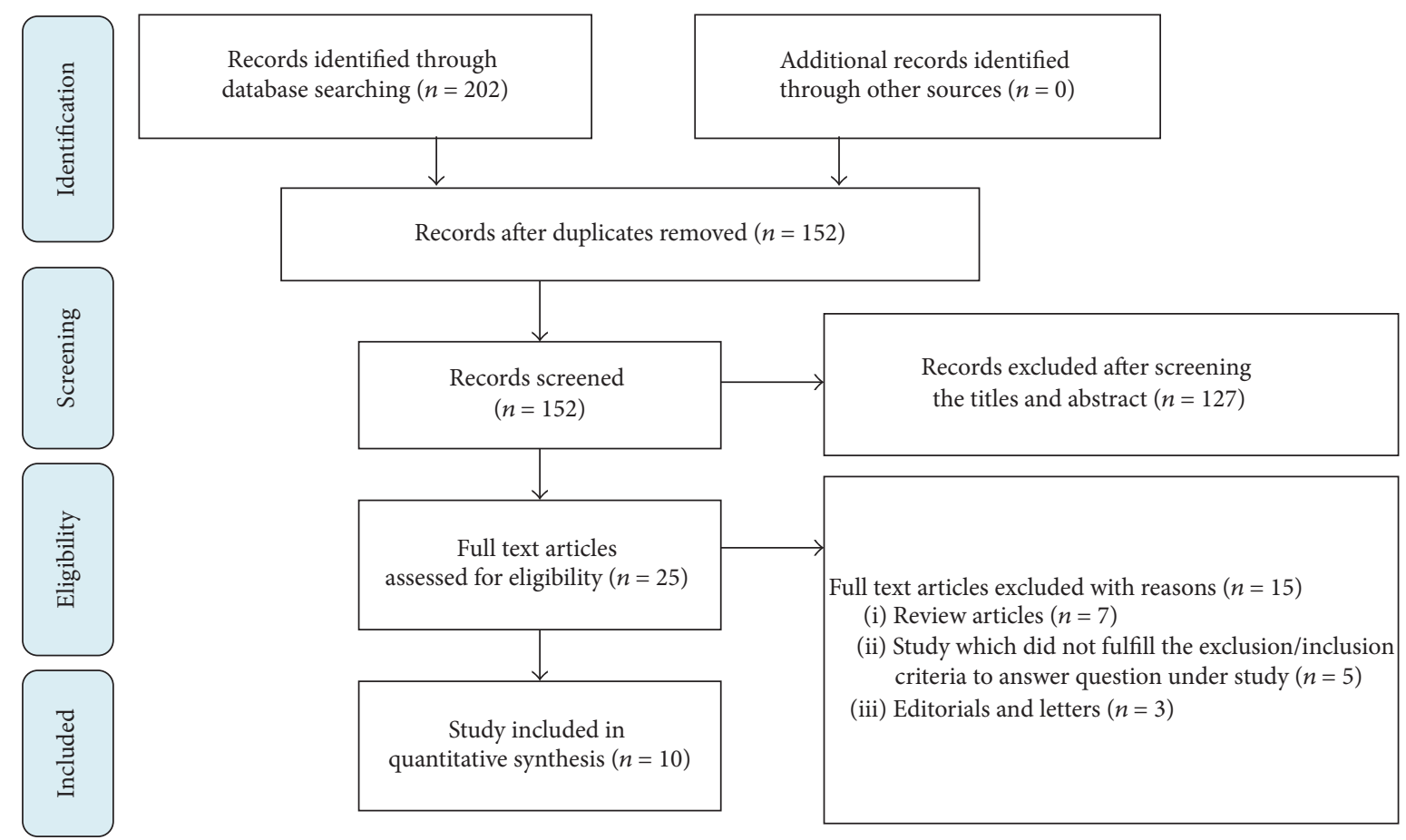

FIGURE 1: Flowchart for trial identification and selection process using the PRISMA statement for systematic review.

Apart from these studies, some studies (cited in the discussion) were also included to support the recommendations of the panel. These articles were referred by individual panelist during the course of discussion and thus used.

3.1. What Is the Optimum Number of Oocytes Associated with the Highest LBR? Sunkara and colleagues in their study demonstrated a firm relationship between the number of oocytes retrieved and LBR. The authors found that LBR increased as the number of oocytes also increased up to 15 , stabilized between 15 and 20 oocytes, and steadily declined beyond 20 oocytes across all female age groups [9]. In their study, the median number of oocytes that were collected was 9 [interquartile range (IQR) 6-13]. Overall, the LBR was $21.3 \%$ [95\% confidence interval (CI): 21.2 to $21.4 \%$ ]. The authors speculated that a higher number of oocytes could lead to a decreased LBR because of raised serum estradiol levels that have a deleterious effect on embryo implantation.

Subsequently, Ji and colleagues retrospectively evaluated 2,455 patients who had undergone IVF treatments in China. The subjects were categorized into four groups on the basis of the number of oocytes retrieved $(0-5,6-10,11-15$, and $>15$ oocytes). In this study, the authors analyzed the relationship between oocyte number and LBR, considering not only fresh cycles but also frozen-thawed cycles, thus allowing estimation of cumulative LBR. In fresh transfers, LBR was optimized when the number of oocytes retrieved was 11-15. The odds of LBR increased from 1.823 (95\% CI: 1.395 to 2.381) in 610 oocyte category to 2.142 (95\% CI: 1.609 to 2.851 ) in $11-15$ oocyte category but decreased in $\geq 16$ oocyte category, being 1.918 (95\% CI: 1.376 to 2.672 ). On combining the fresh and frozen cycles, cumulative LBR per initiated cycle increased with the number of oocytes, but the incidence of moderate and severe ovarian hyperstimulation syndrome (OHSS) also increased. The study suggested that the ideal number of oocytes for achieving highest cumulative LBR (CPR) while avoiding risk of OHSS should range between 6 and 15 [21].

Recently, De Geyter and colleagues compared data generated from over 100,000 IVF cycles performed between 1993 and 2012. The Federal Office of Statistics published this data based on the Swiss database on ART. A panel of experts extracted recommendations from these analyses to improve the current practice, prevent complications related to ART, and further recommend modifications in the current Swiss legislation regarding ART. Among all treated patients, the delivery rates per embryo transfer were dependent on the number of oocytes retrieved $(p<0.0001)$, but among younger patients, this association was less pronounced. It was observed that the number of deliveries was significantly lower if $<5$ oocytes (irrespective of the age groups) were retrieved and did not improve further if $>15$ oocytes were collected (except $>39$ years). The quantity of oocytes retrieved determined the likelihood of delivery, risk of multiple births, and incidence of OHSS. As such, the expert panel concluded that an optimum $\mathrm{COH}$ for the retrieval of 10 to 15 oocytes per treatment cycle should be designed [22].

3.2. Is There a Correlation between Cohort Size and Embryo Quality? Ata et al. analyzed 7,753 embryos of 990 women (<35 years, good ovarian reserve) undergoing IVF by an array-based comparative genomic hybridization (aCGH). In a linear regression analysis, a total of 5,918 cleavage-stage 


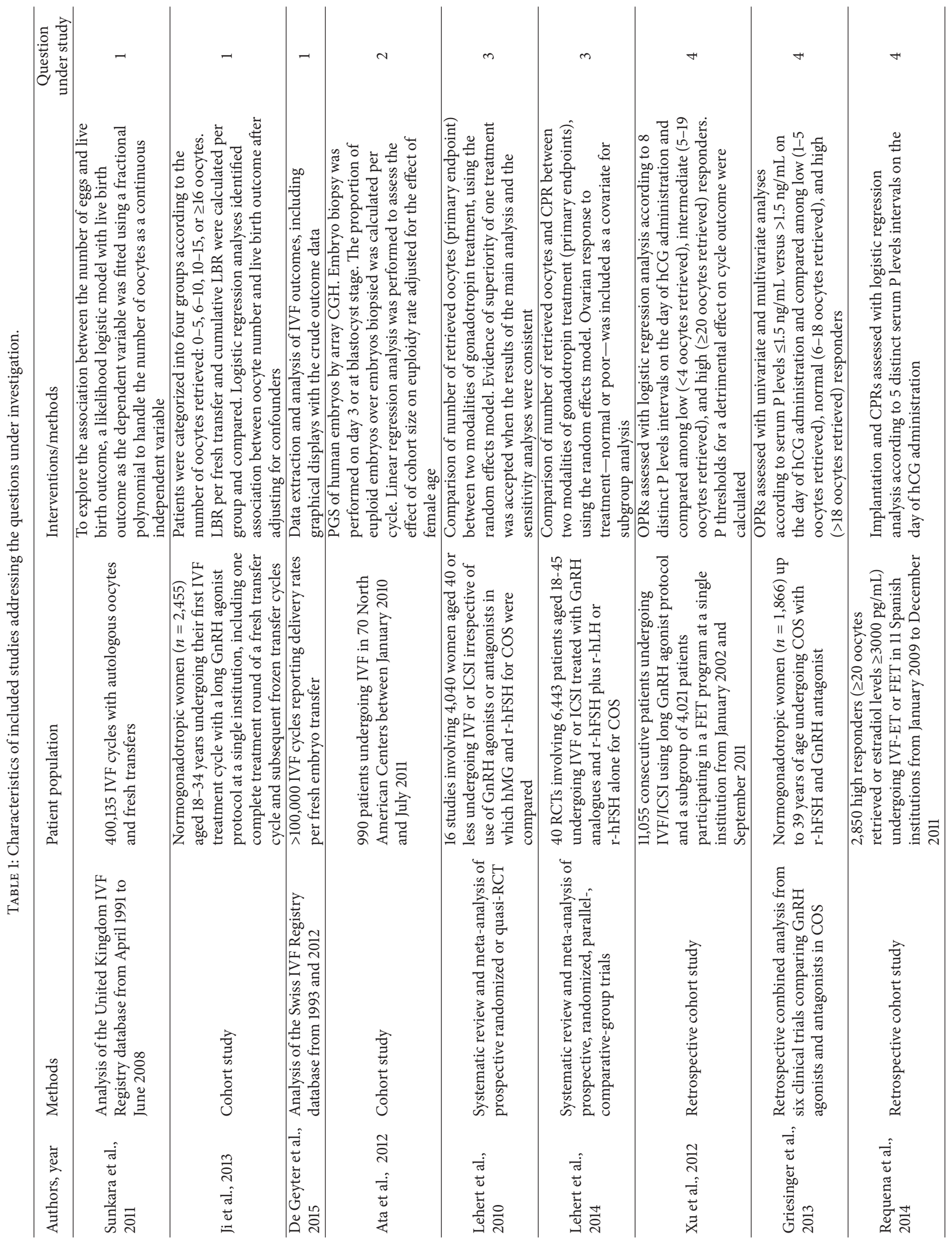




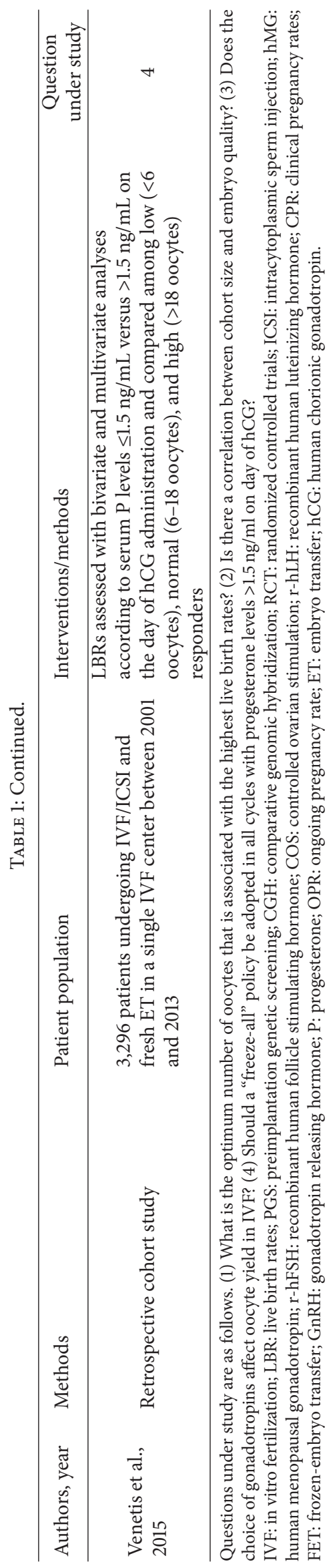


embryos and 1,218 blastocysts obtained from 726 and 218 women, respectively, were analyzed with aCGH. The authors observed that, for each year increase in female age, euploidy rate decreased by 2.9 percentage points in both day 3 and blastocysts (95\% CI: $-3.8 \%$ to $-2.0 \%, p<0.001$ ), and the odds of achieving at least one euploid embryo were decreased as a function of increased female age in both groups (odds ratio (OR) $0.82,95 \%$ CI $0.70-0.94, p=0.006$ ). Interestingly, the quantity of embryos was not associated with the euploidy rate $(B=-0.32 \%, 95 \% \mathrm{CI}:-1.4 \%$ to $0.8 \%)$. Further, the odds of having a minimum of one euploid embryo significantly increased with every additional embryo available for analysis (OR: $1.55,95 \%$ CI: 1.25 to $1.93, p<0.001$ ). The authors concluded that euploidy significantly decreased with age but not with the cohort size [23].

3.3. Do Gonadotropin Isoforms Affect Oocyte Yield in IVF? In a meta-analysis, Lehert and colleagues reported that hMG resulted in significantly fewer oocytes than recombinant human FSH (r-hFSH) (mean 9.4 \pm 6.3 versus 10.9 \pm 6.6 ; mean difference [MD]: $-1.54 ; 95 \% \mathrm{CI}:-2.53$ to $-0.56 ; p<0.0001)$, although a significant heterogeneity was observed among the studies $\left(\mathrm{I}^{2}=63 \%, p=0.0004\right)$. After adjusting for potential confounders, including age, basal FSH, body mass index (BMI), and the number of follicles, the MD was $-2.10(95 \%$ CI: -2.83 to $-1.36 ; p<0.001)$. Further, a higher total dose of hMG was needed during $\mathrm{COH}$ compared with r-hFSH (MD: 235.46 IU [95\% CI: 16.62 to 454.30; $p=0.03$ ]) [24]. The authors estimated ratio of the number of oocytes/1000 IU of gonadotropin dose to be 4.39 and 5.10 for hMG and r$\mathrm{hFSH}$, respectively, with a mean difference favoring $\mathrm{r}-\mathrm{FSH}$ of 0.70 oocytes/1000 IU (95\% CI: 0.10 to $1.30 ; p=0.021$ ). The pregnancy rate did not differ significantly between the two gonadotropin regimens for baseline unadjusted and adjusted estimates.

In a subsequent meta-analysis of 40 trials, including 6,443 women, Lehert and colleagues compared the effect of combining recombinant gonadotropins, FSH and $\mathrm{LH}$, with rhFSH alone on oocyte yield and pregnancy rates. Prospective, parallel, and randomized controlled trials (RCTs) in women aged 18-45 years undergoing IVF, intracytoplasmic sperm injection (ICSI), or both treated with gonadotropin-releasing hormone $(\mathrm{GnRH})$ analogues were included. Overall, no significant difference was observed in the quantity of oocytes retrieved between the $\mathrm{r}-\mathrm{hFSH}+$ recombinant human $\mathrm{LH}$ $(\mathrm{r}-\mathrm{hLH})$ and $\mathrm{r}-\mathrm{hFSH}$ groups (weighted MD -0.03 ; 95\% CI: 0.41 to 0.34 ). Higher clinical pregnancy rates were achieved with the combination of r-hFSH and r-hLH versus r-hFSH alone in the overall population analyzed (risk ratio [RR]: 1.09; 95\% CI: 1.01 to 1.18; intention-to-treat [ITT] population). The RR for the ongoing pregnancy rate (14 studies; RR: 1.14; $95 \%$ CI: 1.05 to 1.25 ) and LBR (8 studies; RR: $1.11 ; 95 \%$ CI: 1.01 to 1.21 ; ITT population) was significant in favor of the gonadotropin combination. However, a nonsignificant benefit for $\mathrm{r}$-hFSH $+\mathrm{r}$-hLH for ongoing pregnancy rate (OPR) (RR: 1.13; 95\% CI: 1.00 to 1.27 ) and LBR (RR: 1.10; 95\% CI: 0.94 to 1.29 ) was observed in normal responders [25].
Data on poor responders (POR) were available from 14 studies in the aforementioned meta-analysis, and a subgroup analysis of this patient population was carried out. Poor responders were defined according to study authors' criteria, and in 10 of the 14 included studies, the definition of POR was aligned with the reported Bologna criteria (European Society of Human Reproduction and Embryology (ESHRE)) [26]. In the overall population of poor responders, a significant high number of oocytes were collected with r-hFSH $+\mathrm{r}-\mathrm{hLH}$ versus $r$-hFSH alone ( $n=1077$; weighted MD: +0.75 oocytes; 95\% CI 0.14 to 1.36 ). A stronger benefit was found in the subgroup of patients who were younger ( $<36$ years of age) poor responders and received GnRH agonist for pituitary desensitization (MD: +1.40 oocytes; $95 \% \mathrm{CI}$ : 0.35 to 2.46 ; $p=0.01)$. The authors also noted a significantly higher clinical pregnancy rate with the combination of recombinant gonadotropins versus r-hFSH alone (RR: 1.30; 95\% CI: 1.01 to 1.67; ITT population). Data about the OPR was available in 11 studies (1043 patients); among these, a significant benefit was observed overall for r-hFSH + r-hLH (RR: 1.36; 95\% CI: 1.04 to 1.79). A nonsignificant benefit for $\mathrm{r}-\mathrm{hFSH}+\mathrm{r}-\mathrm{hLH}$ on $\mathrm{LBR}$ was observed in the subgroup of poor responders (RR: 1.30; 95\% CI 0.95 to 1.78 ) [25].

3.4. Should "Freeze-All" Policy Be Adopted in All Cycles with $P$ Levels $>1.5 \mathrm{ng} / \mathrm{mL}$ on Day of $h C G$ ? Xu et al. reported the association between serum P levels on the day of hCG administration and OPRs in 11,055 women subjected to their first IVF/ICSI cycles. Their study involved different responders undergoing fresh transfers, including high ( $\geq 20$ oocytes; $n=$ 2023 ), poor ( $\leq 4$ oocytes; $n=827$ ), or intermediate $(5-19$ oocytes; $n=8,205$ ) according to the quantity of oocytes harvested, as well as 4,021 women subjected to frozen-thawed embryo transfer (FET) cycles. The mean serum levels of $\mathrm{P}$ on the day of hCG administration were $1.51 \pm 0.51 \mathrm{ng} / \mathrm{mL}$, but they noted a significantly higher serum $\mathrm{P}$ levels in the high ovarian response subgroup than in the intermediate and poor ovarian response subgroups $(1.89 \pm 0.66 \mathrm{ng} / \mathrm{mL}$, $1.47 \pm 0.47 \mathrm{ng} / \mathrm{mL}$, and $1.18 \pm 0.48 \mathrm{ng} / \mathrm{mL}$, respectively; $p<$ $0.001)$. The women in poor ovarian response group had the lowest P levels compared to other groups $(p<0.001)$. In a multivariate logistic regression analysis, the number of oocytes retrieved, total FSH dose, and serum estradiol values were positively associated with increased $\mathrm{P}$ levels on the day of hCG administration. After stratifying patients into eight groups according to serum P levels on the day of hCG administration $(<1.00,1.00-1.25,1.25-1.50,1.50-1.75,1.75-$ $2.00,2.00-2.25,2.25-2.5$, and $>2.5 \mathrm{ng} / \mathrm{mL}$ ), they observed statistically significant reduced OPR according to the ovarian response category. When serum $\mathrm{P}$ was $>2.25 \mathrm{ng} / \mathrm{mL}$, there was a statistically significant reduced OPR (27.5\% and $36.8 \%)$ in high responders (OR: $0.47 ; 95 \% \mathrm{CI}: 0.26$ to 0.85 ), although the fertilization and cleavage rates were not compromised. For the intermediate and poor responders, the threshold levels to discriminate patients with reduced OPR were $1.75 \mathrm{ng} / \mathrm{mL}$ (OR: $0.78 ; 95 \%$ CI: 0.65 to 0.95 ) and $1.5 \mathrm{ng} / \mathrm{mL}$ (OR: 0.36 ; 95\% CI: 0.14 to 0.94 ), respectively. The percentage of subjects with elevated P levels on the day of hCG was $24.2 \%$ 
overall, and $21.5 \%, 25.6 \%$, and $17.0 \%$ in the categories of high, intermediate, and poor responders, respectively. In contrast to fresh cycles, elevated serum P levels did not significantly influence fertilization, cleavage rates, and OPR in FET cycles [27].

Subsequently, Griesinger and colleagues analyzed ovarian response and OPR based on serum P levels $\leq 1.5 \mathrm{ng} / \mathrm{mL}$ and $>1.5 \mathrm{ng} / \mathrm{mL}$ on the day of hCG administration by pooling six trials including 1,866 women subjected to IVF with rhFSH and GnRH antagonist. They observed that women with elevated $\mathrm{P}(>1.5 \mathrm{ng} / \mathrm{mL})$ had a higher ovarian response and higher quantity of oocytes retrieved as compared to women who had $\mathrm{P}<1.5 \mathrm{ng} / \mathrm{mL}(p<0.01)$. The OR of ongoing pregnancy per embryo transfer for $\mathrm{P}$ category $(>1.5 \mathrm{ng} / \mathrm{mL}$ versus $<1.5 \mathrm{ng} / \mathrm{mL}$ ) on the day of hCG was 0.56 (95\% CI: 0.37 to 0.83 ) after adjusting for trial, age, duration of stimulation, quantity of oocytes harvested, and number of transferred embryos. However, no detrimental effect of $\mathrm{P}$ elevation $(>1.5 \mathrm{ng} / \mathrm{mL})$ was observed on OPR (39.2\% versus $42.3 \%)$ in women with high ovarian response ( $>18$ oocytes). The percentage of patients with elevated P levels on the day of hCG was $8.4 \%$ overall, and $4.5 \%, 3.9 \%, 8.3 \%, 12.1 \%$, and $19.0 \%$ in women with $1-5,6-9,10-13,14-18$, and $>18$ oocytes retrieved, respectively [28]. The aforementioned authors evaluated the impact on OPR if the negative effect of P elevations could have been prevented and estimated that it would have theoretically bring about an increase in the overall pregnancy rate of 1.0 percentage points, that is, from $32.5 \%$ to $33.5 \%$ in their studied population.

In another study, Requena et al. evaluated the impact of P levels on the day of hCG on IVF outcomes in 2850 women with high ovarian response undergoing IVF in 11 institutions over a 2-year period. High response was defined as women who had $\geq 20$ oocytes harvested or whose estradiol levels were $\geq 3000 \mathrm{pg} / \mathrm{mL}$. The patients were grouped as per their P levels on the day of hCG as follows: (i) $<0.5 \mathrm{ng} / \mathrm{mL}$, (ii) $0.50-0.70 \mathrm{ng} / \mathrm{mL}$, (iii) $0.71-1.00 \mathrm{ng} / \mathrm{mL}$, (iv) $1.01-1.40 \mathrm{ng} / \mathrm{mL}$, (v) $1.41-1.80 \mathrm{ng} / \mathrm{mL}$, and (vi) $>1.81 \mathrm{ng} / \mathrm{mL}$. They observed an association between elevated $\mathrm{P}$ levels and estradiol levels. Further, no significant differences were noted in the mean $\mathrm{P}$ concentration as a function of the type of gonadotropin used for COH: r-hFSH alone $(n=728, \mathrm{P} 1.06 \mathrm{ng} / \mathrm{mL})$, rhFSH + r-hLH $(n=377, \mathrm{P} 1.01 \mathrm{ng} / \mathrm{mL})$, HP-hMG alone $(n=370 ; \mathrm{P} 1.10 \mathrm{ng} / \mathrm{mL})$, and $\mathrm{r}-\mathrm{hFSH}+\mathrm{HP}-\mathrm{hMG}(n=$ $1375 ; \mathrm{P} 1.30 \mathrm{ng} / \mathrm{mL}$ ). At concentration $>1.8 \mathrm{ng} / \mathrm{mL}$ (but not at $1.5 \mathrm{ng} / \mathrm{mL}$ ), the effect of $\mathrm{P}$ rise on the probability of pregnancy was minimum (OR: $0.73,95 \%$ CI 0.61 to 0.99 ) and negligible on the rates of implantation in patients with a high ovarian response. The authors suggested that, in high responders, $\mathrm{P}$ levels cannot be used to predict clinical outcomes as depicted by the uninformative value in the area under the curve (AUC) derived from receiver operating characteristics (ROC) analysis [14].

Recently, Venetis and colleagues estimated the impact of P levels on the day of hCG on LBR by quantifying the effect of most important known confounders using a multivariate regression analysis. These confounders included age of the women and the total number of oocytes retrieved, quantity of embryos that are transferred and their developmental stage, presence of a minimum one good-quality embryo transferred, BMI of woman, total dosage of FSH used, and the type of $\mathrm{GnRH}$ analogues (agonists versus antagonists) for ovarian stimulation. The authors observed that LBR was similar between cycles with higher and lower $\mathrm{P}$ level $(<1.5 \mathrm{ng} / \mathrm{mL})$ when a bivariate analysis was performed (OR: $0.78,95 \% \mathrm{CI}: 0.56$ to 1.09 ). However, in a multivariable analysis controlling for the effect of the confounders, LBR (OR: $0.68,95 \%$ CI 0.48 to 0.97 ) was significantly decreased in patients with higher P levels on the day of hCG. When the analysis was performed based on quantity of oocytes retrieved, nothing significant could be found in cycles with $<6$ oocytes and $>18$ oocytes. However, a negative impact of P levels on LBR was detected that achieved a statistical significance in normal responders (6-18 oocytes, $n=1770$ cycles) when $0.9 \mathrm{ng} / \mathrm{mL}, 1.2 \mathrm{ng} / \mathrm{mL}$, and $1.5 \mathrm{ng} / \mathrm{mL}$ were used as threshold levels [29]. In this aforementioned study, P elevation $(>1.5 \mathrm{ng} / \mathrm{mL})$ on the day of hCG was observed in 243 cycles (7.4\%, 95\% CI: $6.5 \%-8.3 \%)$. Based on the quantity of oocytes retrieved, the incidence of P elevation $(>1.5 \mathrm{ng} / \mathrm{mL})$ was $1.4 \%$ (95\% CI $0.8 \%$ to $2.5 \%$ ) in patients with $<6$ oocytes, $6.3 \%$ (95\% CI $5.3 \%$ to $7.6 \%)$ in patients with $6-18$ oocytes, and $16.5 \%$ (95\% CI $13.9 \%$ to $19.3 \%)$ in those with $>18$ oocytes.

\section{Discussion}

Despite the notable advancement in ART, practitioners still face many challenges in daily clinical practice to properly individualize $\mathrm{COH}$. While the birth of a healthy offspring is the ultimate goal, treatment strategies, including ovarian stimulation, should be planned to avoid serious complications such as multiple deliveries and OHSS. Although multiple drug options and treatment protocols are currently available, the age of patients pursuing ART is continually rising which poses an additional barrier to success. Discontented with these issues, a panel of ART experts from India was formed to formulate a consensus to answer relevant clinical questions related to optimal personalized outcome in $\mathrm{COH}$. This review focused on four interrelated clinical questions, and the results of this panel meeting demonstrated that a consensus was reached among specialists on how to increase effectiveness of ART with regard to $\mathrm{COH}$ as discussed below (Figure 2).

The first question focused upon the association of the ideal quantity of oocytes and highest LBR. In 2010, a systematic review and meta-analysis of 14 studies that included $>30000$ patients found a robust association between the number of oocytes (OR: 1.04, 95\% CI 1.02 to 1.07) and pregnancy rate in IVF, whereas female age [OR: $0.95,95 \%$ CI 0.94 to 0.96 ] was negatively associated with chance of pregnancy [30]. Unfortunately, the authors did not estimate the optimum number of oocytes to be targeted in a given $\mathrm{COH}$ attempt, which is crucial as too low number might not result in IVF success and too high number might lead to additional risk factors like OHSS.

The answer to the question was provided by two independent studies involving large data registries in the United Kingdom and Switzerland. Sunkara and colleagues reported 


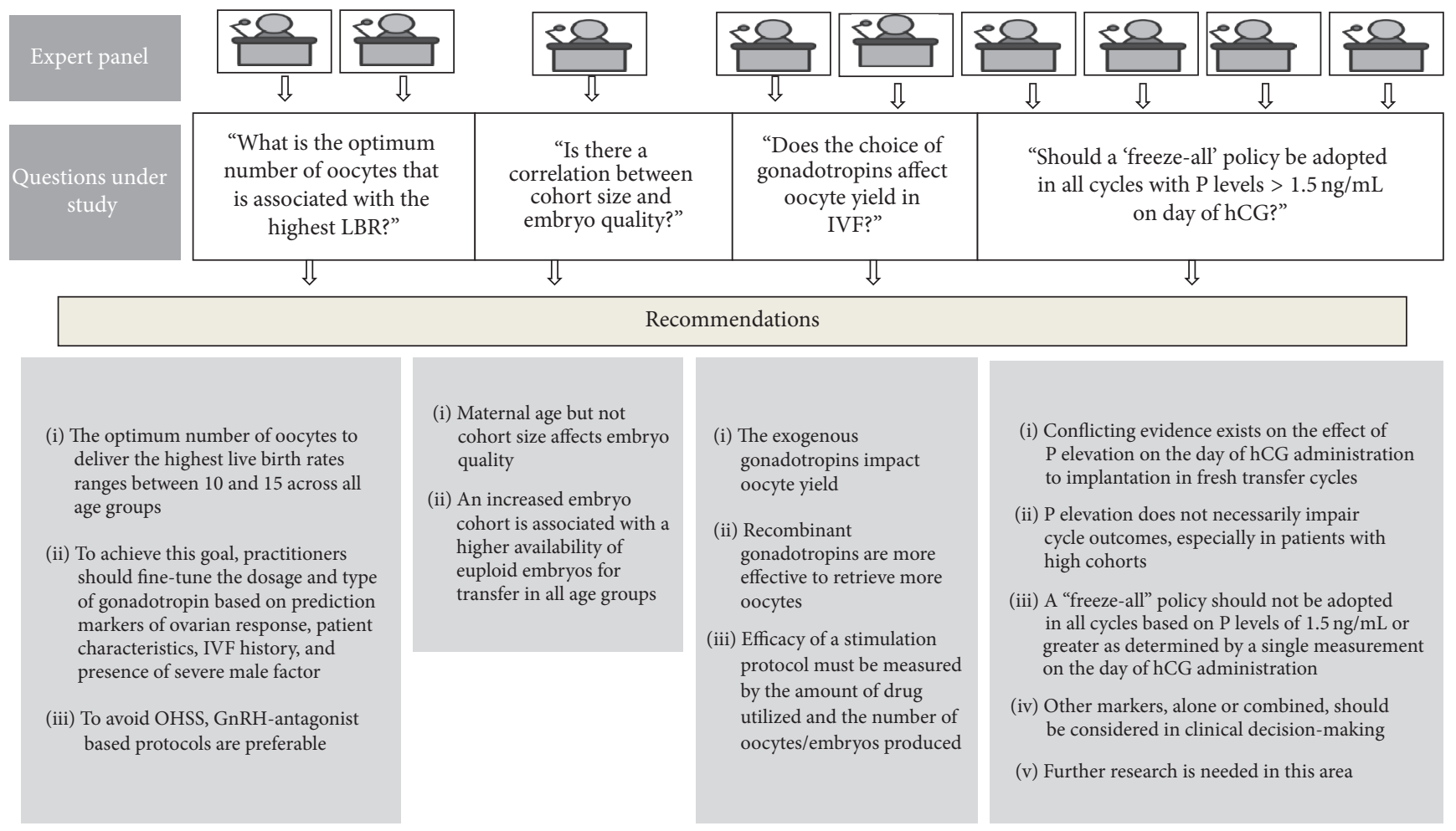

FIGURE 2: Composition of expert panel, questions under study, and recommendations.

that, in different age groups, namely, 18-34, 35-37, 38-39, and $\geq 40$ years, the best chance of live birth in an ART is when the number of eggs retrieved is around 15. In cycles involving fresh embryo transfers, LBR declines with an increase in number of eggs beyond 20 [9]. The LBRs were found to decrease not only with female age, as expected, but also with increasing number of retrieved oocytes $(>20)$. The latter could be due to the harmful effect of elevated serum estradiol levels that in turn affect embryo implantation and increase the risk of OHSS [31-34]. The authors thus demonstrated an association between retrieved oocytes and live birth in a fresh IVF cycle across all age groups, suggesting the quantity of oocytes, in addition to age, to be a reasonable surrogate marker in IVF success. They also generated a nomogram which linked the predicted quantity of oocytes to live birth, likely facilitating the planning of $\mathrm{COH}$ to both optimize outcomes and prevent complications due to production of excessive oocytes. Along the same lines, De Geyter and colleagues found that outcome of ART strongly depend on the female age and number of collected oocytes [22]. It was observed that the quantity of retrieved oocytes significantly affected the delivery rates per fresh embryo transfer $(p<$ $0.0001)$, but this dependence was less important among younger patients. Interestingly, the number of deliveries was significantly lower in each age category if less than five oocytes were harvested. In all the age categories (except $>39$ years), the delivery rates did not improve further, if $>15$ oocytes were collected. Further, a significant rise in the incidence of OHSS was reported if $>14$ oocytes were collected, regardless of age of the patient. Overall, 17.8\% of the embryo transfers among all the initiated treatment cycles were cancelled (irrespective of the age groups), if fewer than 5 but, more frequently, if $\geq 19$ oocytes were harvested. The consensus is also supported by Ji and colleagues, who added by showing that the number of retrieved oocytes is associated with cumulative pregnancy rates, as significantly higher CPR (59\% versus 35\%) has been obtained with 11-15 oocytes retrieved compared with $<6$ oocytes [21].

Collectively, these evidences clearly indicate that the quantity of retrieved oocytes is a positive predictor of live birth in IVF. In addition, increased number of oocytes and embryos will be associated with higher cumulative pregnancy and LBR. Our expert panel concluded that the optimum number of oocytes to deliver highest LBR ranges between 10 and 15, and whenever possible, stimulation protocols should be designed to achieve this goal. There is a high variability in the potency of a woman's ovaries in response ovarian stimulation and thus, the recommended strategy to obtain this optimum number of oocytes would be to tailor the dosage and type of gonadotropin according to the prediction markers of ovarian response to stimulation, particularly antimullerian hormone (AMH) and antral follicle count (AFC), in addition to patient clinical characteristics, previous history of $\mathrm{COH}$ response, and presence of severe male factor infertility [35-40]. To avoid the occurrence of OHSS, we recommended the use of GnRH antagonists in addition to refining the appropriate dosage of gonadotropin. Notwithstanding, it is important to recognize that individual practices may vary depending on the conditions in which the fertility doctor has to work. Whether vitrification technology 
is available, single embryo transfer, or the culture strategy (D3 or blastocyst), might influence the stimulation strategy.

Increased numbers of retrieved oocytes resulting from tailored $\mathrm{COH}$ are important to optimize LBR, as discussed in the previous section. Provided that the embryo quality is critical to IVF success, the next relevant question that the practitioners would like to know is whether an increased oocyte cohort affects the oocyte quality, and consequently, the embryo quality.

Although conventional embryo morphology, including blastomere number and size as well as cytoplasmic fragmentation, still remains the most used criteria for assessing embryo quality, it is limited as a surrogate marker of embryo viability [41]. Poor embryo morphology may reflect oocytes with compromised development competence, but a "so-called" normal embryo may also carry chromosomal abnormalities [42]. In addition, conventional morphological assessment is both subjective and time-dependent and therefore may lead to erroneous discrimination of embryos with the best implantation ability $[41,43,44]$.

Given aneuploidy is the foremost cause of implantation failure, screening for genetic competence of developing embryos remains the gold standard method for assessing embryo quality $[45,46]$. Notwithstanding, this does not mean that an euploid embryo will necessarily implant and develop as some other reasons such as inappropriate endometrial receptivity might also cause implantation failure even in normal embryos [47-50].

Among the methods to assess embryo genetic profile, fluorescence in situ hybridization (FISH), aCGH, and more recently, next generation sequencing (NGS) have been utilized $[51,52]$. Preimplantation genetic screening (PGS) of the embryos has been found to be a successful technique to analyze aneuploidy, specifically in females with advanced maternal age ( $>36$ years) and those with repetitive implantation failures [53-55].

In a study of 1,255 cleavage-stage human embryos using FISH, aneuploidy increased as a function of female age, from $3.1 \%$ in embryos from $20-34$ years old patients to $17 \%$ in patients 40 years or older. But only chromosomes X, Y, 13, 16,18 , and 21 were analyzed, as FISH allows analysis of a limited number of chromosomes [56]. In contrast, aCGH has been shown to be technologically better and more precise technique for PGS than FISH as it can analyze all 24 chromosomes in a single cell with only $1.9 \%$ error rate $[44,46]$. Using this method, Ata and colleagues clearly demonstrated that female age is main factor determining the aneuploidy rate in both cleavage-stage embryos and blastocysts. Interestingly, increased cohorts did not impact embryo euploidy rates, but actually higher number of embryos $(\geq 7)$ were associated with the presence of a minimum of one euploid embryo available for transfer in the vast majority of patients across all age groups [23].

The ability of oocyte to be fertilized is largely determined by morphological and functional changes that are controlled by hormonal events, specifically fluctuation of $\mathrm{LH}$ and FSH, which work synergistically to regulate the follicle development [8]. The biological activity of FSH is decided by the attachment of carbohydrate moieties, forming heterodimers
[8]. However, the degree of gonadotropins' glycosylation is differentially regulated depending on the steroidal status, and therefore, the pattern of circulating FSH during the menstrual cycle is related both to its quantity (concentration) and isoform distribution (quality) [57]. By receptor mediated binding the G protein-coupled receptors present on the granulosa cells (GCs), the gonadotropins containing FSH stimulate the recruitment and growth of early antral follicles $(2-5 \mathrm{~mm}$ in diameter) [8]. In response to this, activation of adenylate cyclase-mediated signal ensues. Following this, the multiple mRNAs are expressed that encode proteins responsible for cell proliferation, differentiation, and function [58]. The relative proportions of isoforms in human $\mathrm{FSH}$ products, either urinary-derived hFSH (u-hFSH) or r-hFSH, depend on the manufacturing process and the origin of the raw material. More acidic isoforms are isolated from urinary products that contain higher numbers of sialic acid residues and have a longer half-life than the more basic isoforms. The more basic isoforms are recombinant molecules that demonstrate higher receptor binding affinity than the more acidic isoforms [59]. On the contrary, increasing the FSH levels in the cells during the first days of stimulation, downregulation of FSH receptors in the granulosa cells has been observed (since the follicular diameter increases $\sim 10 \mathrm{~mm}$ ) [60].

Exogenous gonadotropins are used to stimulate the ovaries to grow follicles producing oocytes. As a result of the specific isoform profile and gonadotropin content, different commercial preparations may deliver different qualitative and/or quantitative signals to the follicle and contribute to different clinical efficacy outcomes [61]. In unselected patients undergoing IVF, an average of three additional oocytes was obtained with recombinant preparations compared with urinary preparations, resulting in more embryos produced with less total gonadotropin dose [24]. In poor responders, fair evidence indicates that addition of $\mathrm{r}-\mathrm{LH}$ to $\mathrm{r}$-hFSH preparations would further improve results, as approximately one additional oocyte was obtained by using this aforementioned drug regimen [24]. In a decision-making case-control matched study on the use of combination protocols that compared a fixed ratio of $2: 1 \mathrm{r}$-hFSH plus $\mathrm{r}$ $\mathrm{hLH}$ or hMG, either alone or in combination with r-hFSH, after downregulation in a long $\mathrm{GnRH}$-agonist protocol in 4,719 women, it was observed that supplementation with $\mathrm{r}$ hLH was significantly more effective. The quantity of oocytes harvested was higher in the r-hFSH and r-hLH combination group [62]. In this study, consumption of r-hFSH was lower $(p<0.001)$ with higher pregnancy rates per cycle $(25.5 \%$ versus $21.5 \% ; p=0.006$; for urinary-derived hMG (u$\mathrm{hMG}$ ) alone and $21.7 \%$ for u-hMG $+\mathrm{r}-\mathrm{hFSH} ; p=0.022$ ) and per embryo transfer $(31.3 \%$ versus $26.0 \%$ for u-hMG alone; $p=0.025$ and $25.6 \%$ for u-hMG + r-hFSH; $p=$ $0.008)$ and implantation per embryo transferred (19\% versus $13.9 \%$ and $13.8 \%$ for $\mathrm{u}-\mathrm{hMG}$ alone and $\mathrm{u}-\mathrm{hMG}+\mathrm{r}-\mathrm{hFSH}$, resp.; both $p<0.001)$ in the group treated with the fixed combination of $\mathrm{r}-\mathrm{hFSH}$ and $\mathrm{r}-\mathrm{hLH}$. As far as pregnancy is concerned, the literature is rich in meta-analyses comparing efficacy of different gonadotropin products (reviewed by Leão and Esteves) [8]. Despite the heterogeneity pertaining 
the different stimulation strategies and fertilization method, the overall conclusion is that both urinary gonadotropins, mainly hMG preparations, and r-FSH have similar efficacy in terms of achieving a pregnancy or live birth per treatment cycle. Collectively, these findings translate two principles on stimulation, namely, the issue that $\mathrm{r}-\mathrm{hFSH}$ is more potent on its receptor than $\mathrm{u}-\mathrm{FSH}$ and the fact that $\mathrm{LH}$ activity $(\mathrm{LH}$ or hCG driven) seems to have a role that affects pregnancy outcome.

Premature $\mathrm{P}$ rise in non-GnRH analogue cycles has been related to poor oocyte maturation, decreased fertilization, and impaired embryo quality [63]. This phenomenon is related to an early preovulatory LH elevation $[64,65]$, which also leads to endometrial asynchrony and may affect implantation and OPR. In contrast, serum P levels mainly reflect $\mathrm{P}$ output of granulosa cells in GnRH agonist and antagonist cycles as the pituitary is suppressed [14, 66, 67].

In fact, prior to ovulation, granulosa cells in the intrafollicular compartment under $\mathrm{LH}$ regulation produce $>95 \% \mathrm{P}$ [68]. In preovulatory follicles, small follicles are "androgenic," while larger follicles are "estrogenic" and "progestogenic." The shift from estrogenic to progestogenic state does not involve increase in the concentration of $\mathrm{P}$ and this shift is consistent with an increased steroidogenesis by granulosa cells as well as an increased LH sensitivity of granulosa cells [69].

$\mathrm{P}$ rise on or before the day of hCG administration occurs in $5-30 \%$ of $\mathrm{COH}$ with $\mathrm{GnRH}$ analogue suppression and has been associated with an increased quantity of retrieved oocytes and the potency of gonadotropin used for $\mathrm{COH}$ [27$29,70]$. In case of recombinants, rise in $\mathrm{P}$ is likely to be more pronounced, as more mature follicles (more granulosa cells) are recruited, while with urinaries, follicle recruitment is less and follicle recruited is a mixture of mature and atretic follicles. Although some authors have suggested that LH activity driven by hCG content in hMG preparations supports the transformation of intrafollicular $\mathrm{P}$ to estradiol, thus increasing endometrium receptivity to embryo implantation, the key enzyme cytochrome 17a-hydroxylaseC17, 20 lyase $(\mathrm{P} 450-17 \alpha)$ that converts $\mathrm{P}$ to estradiol is virtually absent in the intrafollicular compartment $[66,68$, 71]; hence $\mathrm{P}$ conversion to estradiol is negligible in humans [72].

Conflicting views have emerged on the impact of $\mathrm{P}$ rise measured on the day of hCG administration to implantation in fresh transfers. Some researchers have reported that raised $\mathrm{P}$ levels $(>1.5 \mathrm{ng} / \mathrm{mL})$ would be detrimental and thus a "freeze-all" embryos policy should be adopted, while others suggested that elevated P levels are associated with a good ovarian response and no detrimental effect on implantation rates $[13,20,34,73-75]$. The $\mathrm{P}$ threshold in which implantation would be affected is also a matter of debate. Some authors indicated that the chances for a successful pregnancy outcome are decreased if $\mathrm{P}$ levels are $0.8 \mathrm{ng} / \mathrm{mL}$ or greater [75], while others noted that different thresholds should be considered according to the ovarian response [27]. It has also been shown that elevated $\mathrm{P}$ levels are not detrimental to implantation in high responders $[14,28]$. In such patients, it has been hypothesized that good embryo quality counteracts any detrimental effect of elevated P levels to endometrial receptivity [28]. However, in women with poor ovarian response, both factors act synergistically and therefore, pregnancy rates might be reduced [67].

Along the same lines, recent observations have suggested that other markers would better reflect the impact of a $\mathrm{P}$ rise on implantation, including the duration of $\mathrm{P}$ elevation, $\mathrm{P}$ to estradiol ratio, and $\mathrm{P}$ to follicle ratio $[18,76,77]$. In a recent study, Lee and colleagues reported that the clinical pregnancy rate dropped with increase in duration of $\mathrm{P}$ elevation $(39.6 \%$ in women with no $\mathrm{P}$ elevation, $38.9 \%$ with 1 day of $\mathrm{P}$ elevation, $27.3 \%$ with 2 days of $\mathrm{P}$ elevation, and $0 \%$ with 3 days of $\mathrm{P}$ elevation) on or before the day of $\mathrm{LH}$ surge [77]. In another study, Shufaro and colleagues analyzed 8,649 IVF treatments in normal responders and compared the association of total blood P level and calculated progesterone-to-follicle index (PFI; calculated by dividing blood $\mathrm{P}$ to the total number of follicles $\geq 14 \mathrm{~mm}$ ) with the clinical pregnancy rate [18]. They observed that PFI was significantly better correlated with IVF cycle outcome than blood P levels $(p<0.0001)$. Elevated $\mathrm{P}$ levels led to a lower pregnancy rate only if the $\mathrm{P}$ levels were $>93$ percentile. The authors concluded that an elevation in $\mathrm{P}$ levels due to an increased number of follicles is not as important as high PFI, which could negatively affect the pregnancy rate.

In conclusion, there is a lack of clarity among IVF specialists regarding adopting "freeze-all" policy in all cycles with levels of serum $\mathrm{P}>1.5 \mathrm{ng} / \mathrm{mL}$ on day of hCG administration. The $\mathrm{P}$ levels measured in blood represent the sum of $\mathrm{P}$ secreted by multiple follicles. Given the supraphysiological stimulation of granulosa cells, there may be more $\mathrm{P}$ in circulation at the late follicular phase as about $95 \%$ of the circulating $\mathrm{P}$ is produced by GCs in the ovarian intrafollicular compartment. P levels $>1.5 \mathrm{ng} / \mathrm{mL}$ do not necessarily relate to worsening of cycle outcomes, especially in patients with high cohorts. $\mathrm{COH}$ regimens with $\mathrm{LH}$ activity are not useful to reduce serum $\mathrm{P}$ because its conversion to estradiol is negligible owing to a lack of the enzyme that drives this pathway.

The recommendations of the expert panel on all these issues are listed in Figure 1.

\section{Conclusions}

Clinical pregnancy rates are still considered as the primary goal and surrogate marker of IVF success by many practitioners $[2,3]$, despite firm evidence indicating that the most important goal of ART is to attain healthy offspring [78, 79]. Therefore, hormonal stimulation of the ovaries should be refined to improve effectiveness and safety, allowing the creation of longitudinal families by elective transfer of reduced number of embryos [40]. Considering the importance of the number of oocytes on LBR, cumulative pregnancy rate is probably the most relevant pregnancy endpoint in ART.

The optimum number of oocytes that delivers the highest LBR per treatment is 10-15. Maternal age but not cohort 
size affects embryo quality. An increased embryo cohort is associated with the higher availability of euploid embryos for transfer in all age groups. Whenever possible, COS should be designed to achieve these goals. More acidic isoforms are isolated from urinary products that contain higher numbers of sialic acid residues and have a longer half-life than the more basic isoforms. The more basic isoforms are recombinant molecules that demonstrate higher receptor binding affinity than the more acidic isoforms. Different commercial preparations may deliver different qualitative and/or quantitative signals to the follicle and contribute to different clinical efficacy outcomes due to isoform profile and gonadotropin content. Conflicting evidence exists on the effect of $\mathrm{P}$ elevation determined on the day of hCG administration to implantation in fresh transfer cycles. P elevation does not necessarily impair cycle outcomes, especially in patients with high cohorts. Therefore, a "freeze-all" policy should not be adopted in all cycles with serum $\mathrm{P}$ of $1.5 \mathrm{ng} / \mathrm{mL}$ or greater on the day of hCG. However, further research is still needed in this area.

\section{Competing Interests}

The data were assembled, analyzed, and interpreted by a panel of Indian experts in ART. These activities were organized and funded by Merck Serono, India, an affiliate of Merck KGaA, Darmstadt, Germany.

\section{Authors' Contributions}

Baiju Ahemmed, Vani Sundarapandian, Rohit Gutgutia, Sathya Balasubramanyam, Richa Jagtap, Reeta Biliangady, Priti Gupta, Sachin Jadhav, and Ruma Satwik were involved in the discussions during the expert panel meetings, reviewed the manuscript, and provided expert comments and amendments for formulation of the final manuscript. Baiju Ahemmed, Pavitra Raj Dewda, Priti Thakor, and Sandro C. Esteves conceived the study, participated in its design and coordination, primarily searched the literature, and helped to draft the manuscript. Sandro C. Esteves acted as a moderator during the meetings, help formulate the study questions, and reviewed and provided comments in the final version of the manuscript. Pavitra Raj Dewda organized the meetings and help formulate the study questions. All authors read and approved the final manuscript.

\section{Acknowledgments}

The panel of ART experts was organized by Merck Serono, India, represented by Dr. Pavitra Raj Dewda and Dr. Priti Thakor. The authors acknowledge Dr. Shivali Arora of Knowledge Isotopes Pvt. Ltd. (http://www.knowledgeisotopes.com/) for the medical writing support provided in collating and compiling the comments/suggestions from expert panel and revising the manuscript based on their comments. Funding for medical writing support was provided by Merck Serono.

\section{References}

[1] M. Mukherjee and S. B. Nadimipally, "Assisted reproductive technologies in India," Development, vol. 49, no. 4, pp. 128-134, 2006.

[2] J. Boivin, L. Bunting, J. A. Collins, and K. G. Nygren, "International estimates of infertility prevalence and treatment-seeking: potential need and demand for infertility medical care," Human Reproduction, vol. 22, no. 6, pp. 1506-1512, 2007.

[3] N. Malhotra, R. Pai, H. Pai, D. Shah, and M. Bankar, "Assisted reproductive technology in India: a 3 year retrospective data analysis," Journal of Human Reproductive Sciences, vol. 6, no. 4, pp. 235-240, 2013.

[4] P. C. Steptoe and R. G. Edwards, "Birth after the reimplantation of a human embryo," Lancet, vol. 2, no. 8085, p. 366, 1978.

[5] M. H. van der Gaast, M. J. C. Eijkemans, J. B. van der Net et al., "Optimum number of oocytes for a successful first IVF treatment cycle," Reproductive BioMedicine Online, vol. 13, no. 4, pp. 476-480, 2006.

[6] R. G. Edwards, “Test-tube babies, 1981," Nature, vol. 293, no. 5830, pp. 253-256, 1981.

[7] G. B. Inge, P. R. Brinsden, and K. T. Elder, "Oocyte number per live birth in IVF: were Steptoe and Edwards less wasteful?" Human Reproduction, vol. 20, no. 3, pp. 588-592, 2005.

[8] R. D. B. F. Leão and S. C. Esteves, "Gonadotropin therapy in assisted reproduction: an evolutionary perspective from biologics to biotech," Clinics, vol. 69, no. 4, pp. 279-293, 2014.

[9] S. K. Sunkara, V. Rittenberg, N. Raine-Fenning, S. Bhattacharya, J. Zamora, and A. Coomarasamy, "Association between the number of eggs and live birth in IVF treatment: an analysis of 400135 treatment cycles," Human Reproduction, vol. 26, no. 7, pp. 1768-1774, 2011.

[10] S. E. Bulun and E. Y. Adashi, "Physiology and pathology of the female reproductive axis," in Williams Textbook of Endocrinology, chapter 16, Elsevier, 11th edition, 2007.

[11] E. Bosch, I. Valencia, E. Escudero et al., "Premature luteinization during gonadotropin-releasing hormone antagonist cycles and its relationship with in vitro fertilization outcome," Fertility and Sterility, vol. 80, no. 6, pp. 1444-1449, 2003.

[12] T. Harada, S. Yoshida, C. Katagiri et al., "Reduced implantation rate associated with a subtle rise in serum progesterone concentration during the follicular phase of cycles stimulated with a combination of a gonadotrophin-releasing hormone agonist and gonadotrophin," Human Reproduction, vol. 10, no. 5, pp. 1060-1064, 1995.

[13] M. Roque, M. Valle, F. Guimarães, M. Sampaio, and S. Geber, "Freeze-all policy: fresh vs. frozen-thawed embryo transfer," Fertility and Sterility, vol. 103, no. 5, pp. 1190-1193, 2015.

[14] A. Requena, M. Cruz, E. Bosch, M. Meseguer, and J. A. GarciáVelasco, "High progesterone levels in women with high ovarian response do not affect clinical outcomes: A Retrospective Cohort Study," Reproductive Biology and Endocrinology, vol. 12, article 69, 2014.

[15] J. Smitz, A. N. Andersen, P. Devroey, J.-C. Arce, and M. Group, "Endocrine profile in serum and follicular fluid differs after ovarian stimulation with HP-hMG or recombinant FSH in IVF patients," Human Reproduction, vol. 22, pp. 676-687, 2007.

[16] P. Devroey, A. Pellicer, A. Nyboe Andersen, and J.-C. Arce, "A randomized assessor-blind trial comparing highly purified hMG and recombinant FSH in a GnRH antagonist cycle with compulsory single-blastocyst transfer," Fertility and Sterility, vol. 97, no. 3, pp. 561-571, 2012. 
[17] W. Coucke, N. Devleeschouwer, J.-C. Libeer, J. Schiettecatte, M. Martin, and J. Smitz, "Accuracy and reproducibility of automated estradiol-17 $\beta$ and progesterone assays using native serum samples: results obtained in the Belgian external assessment scheme," Human Reproduction, vol. 22, no. 12, pp. 3204-3209, 2007.

[18] Y. Shufaro, O. Sapir, G. Oron et al., "Progesterone-to-follicle index is better correlated with in vitro fertilization cycle outcome than blood progesterone level," Fertility and Sterility, vol. 103, no. 3, pp. 669-674.e3, 2015.

[19] A. N. Andersen, H. Witjes, K. Gordon, and B. Mannaerts, "Predictive factors of ovarian response and clinical outcome after IVF/ICSI following a rFSH/GnRH antagonist protocol with or without oral contraceptive pre-treatment," Human Reproduction, vol. 26, no. 12, pp. 3413-3423, 2011.

[20] O. Hamdine, N. S. Macklon, M. J. C. Eijkemans et al., "Elevated early follicular progesterone levels and in vitro fertilization outcomes: a prospective intervention study and meta-analysis," Fertility and Sterility, vol. 102, no. 2, pp. 448-454, 2014.

[21] J. Ji, Y. Liu, X. H. Tong, L. Luo, J. Ma, and Z. Chen, “The optimum number of oocytes in IVF treatment: an analysis of 2455 cycles in China," Human Reproduction, vol. 28, no. 10, pp. 2728-2734, 2013.

[22] C. De Geyter, P. Fehr, R. Moffat, I. M. Gruber, and M. Von Wolff, "Twenty years' experience with the Swiss data registry for assisted reproductive medicine: outcomes, key trends and recommendations for improved practice," Swiss Medical Weekly, vol. 145, Article ID w14087, 2015.

[23] B. Ata, B. Kaplan, H. Danzer et al., "Array CGH analysis shows that aneuploidy is not related to the number of embryos generated," Reproductive BioMedicine Online, vol. 24, no. 6, pp. 614-620, 2012.

[24] P. Lehert, J. C. Schertz, and D. Ezcurra, "Recombinant human follicle-stimulating hormone produces more oocytes with a lower total dose per cycle in assisted reproductive technologies compared with highly purified human menopausal gonadotrophin: a meta-analysis," Reproductive Biology and Endocrinology, vol. 8, article 112, 2010.

[25] P. Lehert, E. M. Kolibianakis, C. A. Venetis et al., "Recombinant human follicle-stimulating hormone ( $\mathrm{r}-\mathrm{hFSH}$ ) plus recombinant luteinizing hormone versus $\mathrm{r}$-hFSH alone for ovarian stimulation during assisted reproductive technology: systematic review and meta-analysis," Reproductive Biology and Endocrinology, vol. 12, no. 1, article 17, 2014.

[26] A. P. Ferraretti, A. La Marca, B. C. J. M. Fauser, B. Tarlatzis, G. Nargund, and L. Gianaroli, "ESHRE consensus on the definition of 'poor response to ovarian stimulation for in vitro fertilization: the Bologna criteria," Human Reproduction, vol. 26, no. 7, pp. 1616-1624, 2011.

[27] B. Xu, Z. Li, H. Zhang et al., "Serum progesterone level effects on the outcome of in vitro fertilization in patients with different ovarian response: an analysis of more than 10,000 cycles," Fertility and Sterility, vol. 97, no. 6, pp. 1321.e4-1327.e4, 2012.

[28] G. Griesinger, B. Mannaerts, C. Y. Andersen, H. Witjes, E. M. Kolibianakis, and K. Gordon, "Progesterone elevation does not compromise pregnancy rates in high responders: a pooled analysis of in vitro fertilization patients treated with recombinant follicle-stimulating hormone/gonadotropin-releasing hormone antagonist in six trials," Fertility and Sterility, vol. 100, no. 6, pp. 1622.e3-1628.e3, 2013.

[29] C. A. Venetis, E. M. Kolibianakis, J. K. Bosdou et al., "Estimating the net effect of progesterone elevation on the day of hCG on live birth rates after IVF: a cohort analysis of 3296 IVF cycles," Human Reproduction, vol. 30, no. 3, pp. 684-691, 2015.

[30] L. L. van Loendersloot, M. van Wely, J. Limpens, P. M. M. Bossuyt, S. Repping, and F. van der Veen, "Predictive factors in in vitro fertilization (IVF): a systematic review and metaanalysis," Human Reproduction Update, vol. 16, no. 6, Article ID dmq015, pp. 577-589, 2010.

[31] D. Valbuena, J. Martin, J. L. De Pablo, J. Remohí, A. Pellicer, and C. Simón, "Increasing levels of estradiol are deleterious to embryonic implantation because they directly affect the embryo," Fertility and Sterility, vol. 76, no. 5, pp. 962-968, 2001.

[32] M. F. M. Mitwally, H. S. Bhakoo, K. Crickard, M. W. Sullivan, R. E. Batt, and J. Yeh, "Estradiol production during controlled ovarian hyperstimulation correlates with treatment outcome in women undergoing in vitro fertilization-embryo transfer," Fertility and Sterility, vol. 86, no. 3, pp. 588-596, 2006.

[33] T.-H. Lee, C.-H. Liu, C.-C. Huang et al., "Serum anti-müllerian hormone and estradiol levels as predictors of ovarian hyperstimulation syndrome in assisted reproduction technology cycles," Human Reproduction, vol. 23, no. 1, pp. 160-167, 2008.

[34] A. N. Andersen, "Lack of association between endogenous LH and pregnancy in GnRH antagonist protocols," Reproductive BioMedicine Online, vol. 23, no. 6, pp. 692-694, 2011.

[35] F. J. Broekmans, J. Kwee, D. J. Hendriks, B. W. Mol, and C. B. Lambalk, "A systematic review of tests predicting ovarian reserve and IVF outcome," Human Reproduction Update, vol. 12, no. 6, pp. 685-718, 2006.

[36] A. La Marca, S. Giulini, A. Tirelli et al., "Anti-Müllerian hormone measurement on any day of the menstrual cycle strongly predicts ovarian response in assisted reproductive technology," Human Reproduction, vol. 22, no. 3, pp. 766-771, 2007.

[37] S. L. Broer, B. W. J. Mol, D. Hendriks, and F. J. M. Broekmans, "The role of antimullerian hormone in prediction of outcome after IVF: comparison with the antral follicle count," Fertility and Sterility, vol. 91, no. 3, pp. 705-714, 2009.

[38] P. Ocal, S. Sahmay, M. Cetin, T. Irez, O. Guralp, and I. Cepni, "Serum anti-Müllerian hormone and antral follicle count as predictive markers of OHSS in ART cycles," Journal of Assisted Reproduction and Genetics, vol. 28, no. 12, pp. 1197-1203, 2011.

[39] E. Anckaert, J. Smitz, J. Schiettecatte, B. M. Klein, and J.-C. Arce, "The value of anti-Mllerian hormone measurement in the long $\mathrm{GnRH}$ agonist protocol: association with ovarian response and gonadotrophin-dose adjustments," Human Reproduction, vol. 27, no. 6, pp. 1829-1839, 2012.

[40] S. C. Esteves, C. Prudencio, B. Seol, S. Verza Jr., C. Knoedler, and A. Agarwal, "Comparison of sperm retrieval and reproductive outcome in azoospermic men with testicular failure and obstructive azoospermia treated for infertility," Asian Journal of Andrology, vol. 16, no. 4, pp. 602-606, 2014.

[41] ALPHA Scientists in Reproductive Medicine and ESHRE Special Interest Group Embryology, "Istanbul consensus workshop on embryo assessment: proceedings of an expert meeting," Reprod Biomed Online, vol. 22, pp. 632-646, 2011.

[42] S. Munne, M. Alikani, G. Tomkin, J. Grifo, and J. Cohen, "Embryo morphology, developmental rates, and maternal age are correlated with chromosome abnormalities," Fertility and Sterility, vol. 64, no. 2, pp. 382-391, 1995.

[43] F. Devreker, E. Pogonici, V. De Maertelaer, P. Revelard, M. Van Den Bergh, and Y. Englert, "Selection of good embryos for transfer depends on embryo cohort size: implications for the 'mild ovarian stimulation' debate," Human Reproduction, vol. 14, no. 12, pp. 3002-3008, 1999. 
[44] Z. Yang, J. Liu, G. S. Collins et al., "Selection of single blastocysts for fresh transfer viastandard morphology assessment alone and witharray cgh for good prognosis ivf patients: resultsfrom a randomized pilot study," Molecular Cytogenetics, vol. 5, article no. $24,2012$.

[45] S. Munné, J. Ary, C. Zouves et al., "Wide range of chromosome abnormalities in the embryos of young egg donors," Reproductive BioMedicine Online, vol. 12, no. 3, article 2050, pp. 340-346, 2006.

[46] J. F. C. Chow, W. S. B. Yeung, E. Y. L. Lau, V. C. Y. Lee, E. H. Y. Ng, and P.-C. Ho, "Array comparative genomic hybridization analyses of all blastomeres of a cohort of embryos from young IVF patients revealed significant contribution of mitotic errors to embryo mosaicism at the cleavage stage," Reproductive Biology and Endocrinology, vol. 12, p. 105, 2014.

[47] K. Diedrich, B. C. J. M. Fauser, P. Devroey, and G. Griesinger, "The role of the endometrium and embryo in human implantation," Human Reproduction Update, vol. 13, no. 4, pp. 365-377, 2007.

[48] D. D. Carson, E. Lagow, A. Thathiah et al., "Changes in gene expression during the early to mid-luteal (receptive phase) transition in human endometrium detected by high-density microarray screening," Molecular Human Reproduction, vol. 8, no. 9, pp. 871-879, 2002.

[49] B. A. Lessey, "Two pathways of progesterone action in the human endometrium: implications for implantation and contraception," Steroids, vol. 68, no. 10-13, pp. 809-815, 2003.

[50] W.-G. Ma, H. Song, S. K. Das, B. C. Paria, and S. K. Dey, "Estrogen is a critical determinant that specifies the duration of the window of uterine receptivity for implantation," Proceedings of the National Academy of Sciences of the United States of America, vol. 100, no. 5, pp. 2963-2968, 2003.

[51] S. Munné, D. Wells, and J. Cohen, “Technology requirements for preimplantation genetic diagnosis to improve assisted reproduction outcomes," Fertility and Sterility, vol. 94, no. 2, pp. 408430, 2010.

[52] H. Zheng, H. Jin, L. Liu, J. Liu, and W.-H. Wang, "Application of next-generation sequencing for 24-chromosome aneuploidy screening of human preimplantation embryos," Molecular Cytogenetics, vol. 8, article 38, 2015.

[53] L. Rodrigo, V. Peinado, E. Mateu et al., "Impact of different patterns of sperm chromosomal abnormalities on the chromosomal constitution of preimplantation embryos," Fertility and Sterility, vol. 94, no. 4, pp. 1380-1386, 2010.

[54] R. T. Scott Jr., K. M. Upham, E. J. Forman et al., "Blastocyst biopsy with comprehensive chromosome screening and fresh embryo transfer significantly increases in vitro fertilization implantation and delivery rates: a randomized controlled trial," Fertility and Sterility, vol. 100, no. 3, pp. 697-703, 2013.

[55] P. Donoso, C. Staessen, B. C. J. M. Fauser, and P. Devroey, "Current value of preimplantation genetic aneuploidy screening in IVF," Human Reproduction Update, vol. 13, no. 1, pp. 15-25, 2007.

[56] C. Márquez, M. Sandalinas, M. Bahçe, M. Al ikani, and S. Munné, "Chromosome abnormalities in 1255 cleavage-stage human embryos," Reproductive BioMedicine Online, vol. 1, no. 1, pp. 17-26, 2000.

[57] B. J. Arey and F. J. López, "Are circulating gonadotropin isoforms naturally occurring biased agonists? Basic and therapeutic implications," Reviews in Endocrine and Metabolic Disorders, vol. 12, no. 4, pp. 275-288, 2011.
[58] S. Assou, I. Boumela, D. Haouzi et al., "Dynamic changes in gene expression during human early embryo development: from fundamental aspects to clinical applications," Human Reproduction Update, vol. 17, no. 2, pp. 272-290, 2011.

[59] C. Y. Andersen and D. Ezcurra, "What is the clinical relevance of follicle-stimulating hormone isoforms in fertility treatment?" Reproductive Biology Insights, vol. 4, no. 1, pp. 1-10, 2011.

[60] J. V. Jeppesen, S. G. Kristensen, M. E. Nielsen et al., "LHreceptor gene expression in human granulosa and cumulus cells from antral and preovulatory follicles," Journal of Clinical Endocrinology \& Metabolism, vol. 97, no. 8, pp. E1524-E1531, 2012.

[61] C. Bergh, C. M. Howles, K. Borg et al., "Recombinant human follicle stimulating hormone (r-hFSH; Gonal-F) versus highly purified urinary FSH (Metrodin HP): results of a randomized comparative study in women undergoing assisted reproductive techniques," Human Reproduction, vol. 12, no. 10, pp. 2133-2139, 1997.

[62] K. F. Bühler and R. Fischer, "Recombinant human LH supplementation versus supplementation with urinary hCG-based LH activity during controlled ovarian stimulation in the long GnRH-agonist protocol: a matched casecontrol study," Gynecological Endocrinology, vol. 28, no. 5, pp. 345-350, 2012.

[63] W. Schoolcraft, E. Sinton, T. Schlenker, D. Huynh, F. Hamilton, and D. R. Meldrum, "Lower pregnancy rate with premature luteinization during pituitary suppression with leuprolide acetate," Fertility and Sterility, vol. 55, no. 3, pp. 563-566, 1991.

[64] J. S. Younis, A. Simon, and N. Laufer, "Endometrial preparation: lessons from oocyte donation," Fertility and Sterility, vol. 66, no. 6, pp. 873-884, 1996.

[65] R. Fanchin, C. Righini, F. Olivennes, A. L. Ferreira, D. De Ziegler, and R. Frydman, "Consequences of premature progesterone elevation on the outcome of in vitro fertilization: insights into a controversy," Fertility and Sterility, vol. 68, no. 5, pp. 799805, 1997.

[66] C. D. Smyth, F. Miro, P. F. Whitelaw, C. M. Howles, and S. G. Hillier, "Ovarian thecal/interstitial androgen synthesis is enhanced by a follicle-stimulating hormone-stimulated paracrine mechanism," Endocrinology, vol. 133, no. 4, pp. 15321538, 1993.

[67] J. S. Younis, "Elevated P level on the day of hCG administration is related to FSH dose: is it the whole truth?" Human Reproduction, vol. 26, no. 2, pp. 498-499, 2011.

[68] C. Y. Andersen and D. Ezcurra, "Human steroidogenesis: implications for controlled ovarian stimulation with exogenous gonadotropins," Reproductive Biology and Endocrinology, vol. 12, article 128, 2014.

[69] L. Westergaard, I. J. Christensen, and K. P. Mcnatty, "Steroid levels in ovarian follicular fluid related to follicle size and health status during the normal menstrual cycle in women," Human Reproduction, vol. 1, no. 4, pp. 227-232, 1986.

[70] A. Requena, M. Cruz, F. J. Ruiz, and J. A. García-Velasco, "Endocrine profile following stimulation with recombinant follicle stimulating hormone and luteinizing hormone versus highly purified human menopausal gonadotropin," Reproductive Biology and Endocrinology, vol. 12, article 10, 2014.

[71] L. L. Thuesen, A. Loft, A. N. Egeberg, J. Smitz, J. H. Petersen, and A. Nyboe Andersen, "A randomized controlled doseresponse pilot study of addition of hCG to recombinant FSH during controlled ovarian stimulation for in vitro fertilization," Human Reproduction, vol. 27, no. 10, pp. 3074-3084, 2012. 
[72] D. Ezcurra and P. Humaidan, "A review of luteinising hormone and human chorionic gonadotropin when used in assisted reproductive technology," Reproductive Biology \& Endocrinology, vol. 12, article 95, 2014.

[73] C. R. Givens, E. D. Schriock, P. V. Dandekar, and M. C. Martin, "Elevated serum progesterone levels on the day of human chorionic gonadotropin administration do not predict outcome in assisted reproduction cycles," Fertility and Sterility, vol. 62, no. 5, pp. 1011-1017, 1994.

[74] M. I. Abuzeid and M. A. Sasy, "Elevated progesterone levels in the late follicular phase do not predict success of in vitro fertilization-embryo transfer," Fertility and Sterility, vol. 65, no. 5, pp. 981-985, 1996.

[75] C. A. Venetis, E. M. Kolibianakis, J. K. Bosdou, and B. C. Tarlatzis, "Progesterone elevation and probability of pregnancy after IVF: a systematic review and meta-analysis of over 60000 cycles," Human Reproduction Update, vol. 19, no. 5, pp. 433-457, 2013.

[76] T.-H. Lai, F.-K. Lee, T.-K. Lin et al., "An increased serum progesterone-to-estradiol ratio on the day of human chorionic gonadotropin administration does not have a negative impact on clinical pregnancy rate in women with normal ovarian reserve treated with a long gonadotropin releasing hormone agonist protocol," Fertility and Sterility, vol. 92, no. 2, pp. 508514, 2009.

[77] V. C. Y. Lee, R. H. W. Li, J. Chai et al., "Effect of preovulatory progesterone elevation and duration of progesterone elevation on the pregnancy rate of frozen-thawed embryo transfer in natural cycles," Fertility and Sterility, vol. 101, no. 5, pp. 12881293, 2014.

[78] R. Mansour, O. Ishihara, G. D. Adamson et al., "International committee for monitoring assisted reproductive technologies world report: assisted reproductive technology 2006," Human Reproduction, vol. 29, no. 7, pp. 1536-1551, 2014.

[79] Centers for Disease Control and Prevention (CDC), "U.S. Department of Health and Human Services, Assisted Reproduction Technology Success Rates (2012), National Summary and Fertility Clinic Reports," https://www.cdc .gov/art/pdf/2012-report/national-summary/art_2012_national_ summary_report.pdf. 


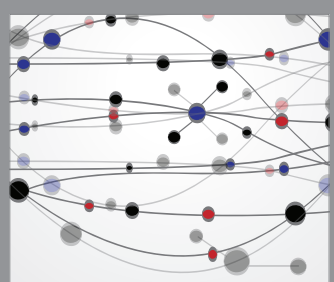

The Scientific World Journal
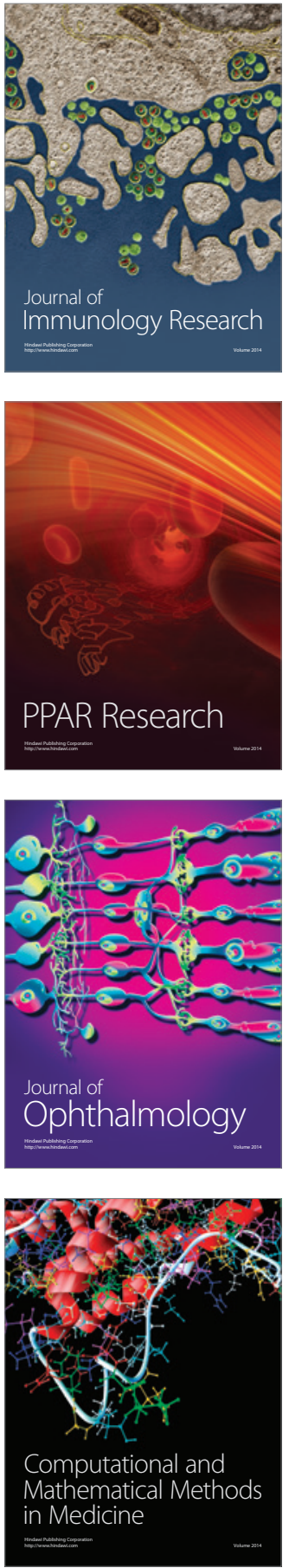

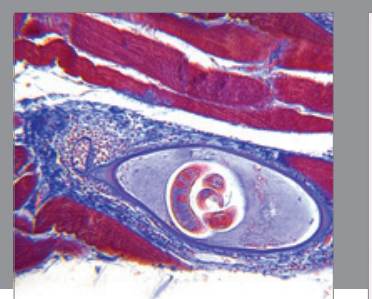

Gastroenterology Research and Practice
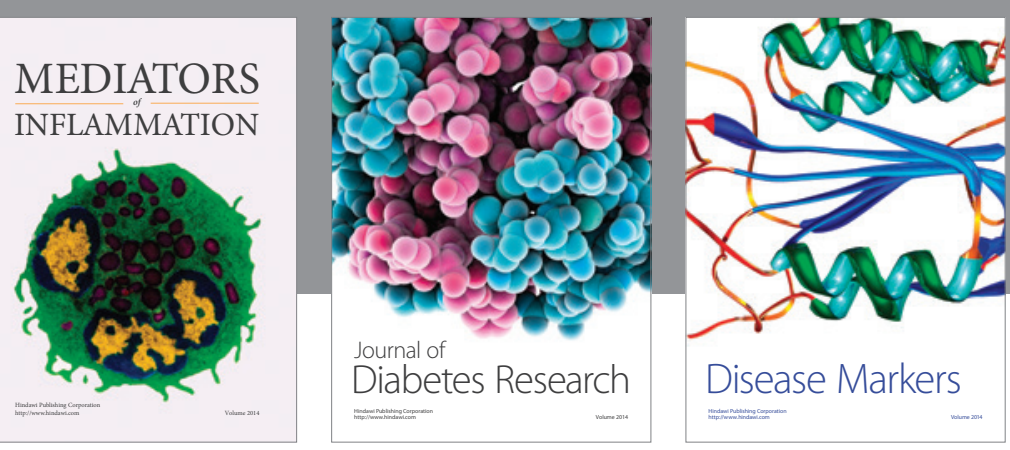

Disease Markers

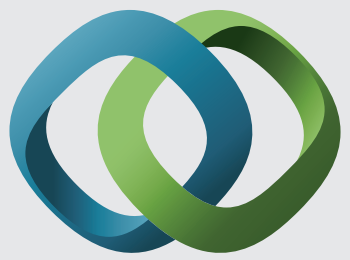

\section{Hindawi}

Submit your manuscripts at

https://www.hindawi.com
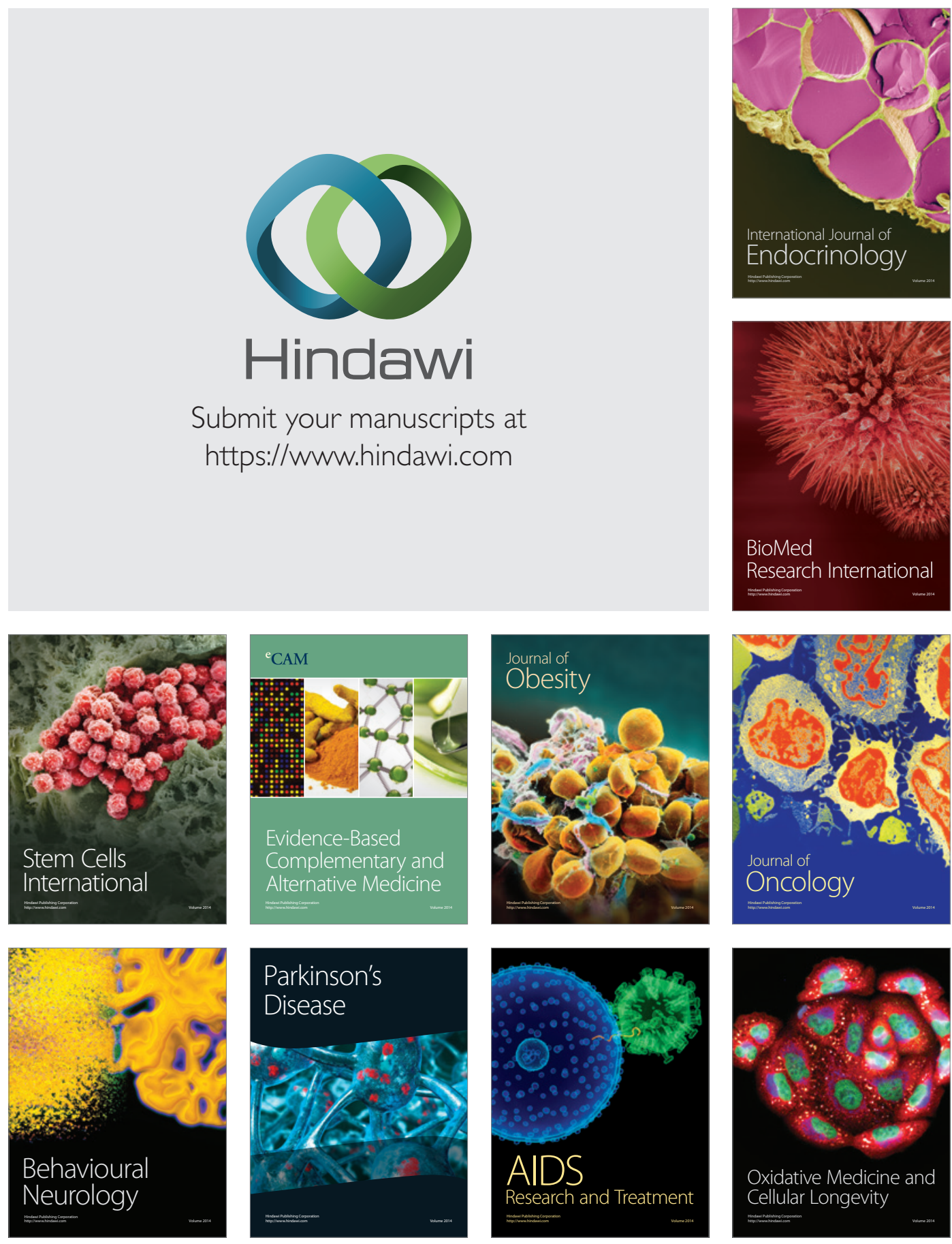